\title{
Hexanal Gas Detection Using Chitosan Biopolymer as Sensing Material at Room Temperature
}

\author{
Devi Shantini, ${ }^{1}$ Irwana Nainggolan, ${ }^{1}$ Tulus Ikhsan Nasution, ${ }^{2}$ Mohd Nazree Derman, \\ Roshida Mustaffa, ${ }^{1}$ and Nur Zuraihan Abd Wahab ${ }^{1}$ \\ ${ }^{1}$ School of Materials Engineering, Universiti Malaysia Perlis, 01000 Kangar, Perlis, Malaysia \\ ${ }^{2}$ Physics Department, Faculty of Mathematic and Natural Science, University of Sumatera Utara, Medan, \\ Sumatera Utara 20155, Indonesia
}

Correspondence should be addressed to Irwana Nainggolan; irwana@unimap.edu.my

Received 30 August 2015; Revised 30 November 2015; Accepted 13 December 2015

Academic Editor: Elisabetta Comini

Copyright (c) 2016 Devi Shantini et al. This is an open access article distributed under the Creative Commons Attribution License, which permits unrestricted use, distribution, and reproduction in any medium, provided the original work is properly cited.

\begin{abstract}
Hexanal was identified as one of the major volatile gases which are produced in degraded dairy products and wood industries. Therefore, preliminary study on hexanal gas detection with the laboratory scale was carried out in this paper. Electrical testing with chitosan as a sensing material to sense hexanal gas in low concentration was carried out at room temperature. Chitosan sensor was fabricated by using electrochemical deposition technique to form active sensing layer. The response of the chitosan film sensor (CFS) towards hexanal was tested via electrical testing by exposing different hexanal concentrations ranging between 20 ppm, 100 ppm, $200 \mathrm{ppm}$, and $300 \mathrm{ppm}$ using air as a carrier gas. Sensing properties of the CFS toward hexanal exposure including responsibility, recovery, repeatability, stability, and selectively were studied. Overall, our result suggested that hexanal sensor based on chitosan was able to perform well at room temperature demonstrated by good response, good recovery, good repeatability, good stability, and good selectively. This simple and low cost sensor has high potential to be utilized in early quality degradation detection in dairy products and can be used to monitor the level of hexanal exposure in wood industries.
\end{abstract}

\section{Introduction}

Hexanal has been utilized in various fields including organic synthesis, rubber, paint, and additive in foods [1]. Besides that, hexanal can be also formed in food especially milk powder when it spoiled or degraded due to oxidation upon storage [2]. It was found that milk powder tends to release around $20 \mathrm{ppm}$ of hexanal in 6 months of storage. In addition, hexanal was also identified as a major emitted gas from wood pellets stored in industrial warehouse. It was reported that hexanal with concentration of approximately $24 \mathrm{ppm}$ is being released in wood pellets due to autooxidation [3]. Basically, inhalation and skin uptake were the main routes for hexanal to enter the human body. Hexanal has cytotoxic potential but only in relatively high doses. A study showed that $10 \mathrm{ppm}$ hexanal exposure can lead to eye discomfort [1]. Modern analytical method which is gas chromatography has been used for hexanal detection especially in milk powder $[2,4]$. However, this type of equipment cannot be used for line detecting pollutant due to its large size, heavy, high cost, and time-consuming operation. Thus, it is important to develop viable low cost and user friendly gas sensor for low concentration hexanal gas detection especially in food and wood industries. Thus, new method and material have been employed for hexanal detection recently.

As reported in literature, there were only few articles reported on hexanal detection. The first paper reported that lutetium bisphthalocyanine was utilized as a sensing material where it was developed by using Langmuir-Blodgett technique and exposed to hexanal using air as carrier [5]. However, the paper did not have much explanation on sensing properties of the sensor upon various hexanal gas concentrations. There is another report where a single-walled nanotube was used as a sensing material [6]. In this report, carbon nanotube has been fabricated via spray deposition method. The response of the sensor was measured by immersing the sensor directly into hexanal. Up until now, there is no report 
on utilization of chitosan as sensing material. Thus, combination of chitosan as new sensing material and electrochemical deposition as new fabrication method for hexanal gas detection was attempted in lab scale at first.

The reason for selection of chitosan as sensing material is due to its unique properties. Chitosan is one of the conducting polymers that received a great recognition, being used in various fields [7, 8]. On top of all, chitosan has the promising characteristics including being abundantly available, low in cost, and biodegradable and having excellent film forming ability and nontoxic properties that make it applicable to electrochemical sensors, biosensors, gas sensors, and volatile organic compound sensors [9-15]. Chitosan can be attained by modifying seafood waste such as crab and shrimp which is a unique material that is well suited for microdevices especially sensors due to its potential to be selectivity deposited using electrochemical deposition method because of high density of amine groups [16].

Meanwhile, electrochemical deposition method was selected for sensor fabrication since it offers some advantages such as higher deposition rate, shorter processing time, and low cost.

The aim of this study is to investigate the sensing performance of chitosan film sensor upon hexanal gas exposure. The sensing properties of the chitosan film sensor including response, response time, recovery time, repeatability, stability, lifetime, and selectivity were studied by exposing the sensor to hexanal with air as carrier gas. Besides that, the characterizations of the chitosan film sensor were done using Fourier Transform Infrared Spectroscopy (FTIR) and Atomic Force Microscopy (AFM).

\section{Experimental}

2.1. Materials. Chitosan powder with a deacetylation degree of $75 \%-85 \%$ was purchased from Sigma-Aldrich Ltd. Meanwhile, acetic acid with $99.9 \%$ purity from HmbG was used as solvent in this research. Hexanal from Merck was used for sensing studies in this research.

2.2. Characterization Tools. There were few types of equipment which have been used in this study. First of all, the functional group characterization of the chitosan film was done by using ATR method with Fourier Transform Infrared Spectroscopy (Perkin Elmer RX1 FT-IR). Besides that, the surface structure of the film was analysed by Atomic Force Microscope (AFM) with Nanoscope IV/Multimode, Digital Instrument.

2.3. Chitosan Film Sensor (CFS) Fabrication. The procedure was started with the chitosan solution gel preparation. Chitosan solution gel was prepared by dissolving chitosan powder in $2 \%$ acetic acid and stirred using a magnetic stirrer with rotation speed of $300 \mathrm{rpm}$ for 24 hours at room temperature on hot plate (WiseStir MSH-30D). In this study, chitosan with concentration of $1.75 \%$ was used. CFS was developed by electrochemical deposition process where two pieces of copper patterned substrate were dipped into the chitosan solution gel and subjected to the constant voltage of $2.5 \mathrm{~V}$ and electrochemical deposition time of 6 minutes to attract the chitosan molecules to stick on the surface of the cathode side copper electrode. After the deposition, the films were annealed in vacuum oven at $100^{\circ} \mathrm{C}$ for 30 minutes to remove excessive moisture in the films.

2.4. Hexanal Gas Sensing Studies. The vapour sensing characteristics of the chitosan film sensor (CFS) towards hexanal gas were investigated at room temperature by recording their electrical responses. CFS was placed in a sealed chamber where it was connected with power supply and multimeter with the input voltage of $1.5 \mathrm{~V}$ as illustrated in Figure 1. The left sensor stabilized for 5 minutes at first. It was followed by exposing the sensing film alternately to both hexanal and dry air for 5 minutes, respectively. The known volume of hexanal in microliter was injected into different chamber and left to vaporize with the aid of a pump. After that, hexanal vapour was exposed to the sealed testing chamber. The exposure of hexanal vapour was carried out for 5 minutes. It was followed by recovering the sensor where it was done by exposure to dry air for 5 minutes as well. The readings of output voltage were taken for every 30 seconds for 5 minutes for both hexanal and dry air, respectively, using multimeter (SANWA CD771). The exposures of both hexanal and dry air were repeated for three times alternately. The sensor response, $R$, was calculated as follows:

$$
R=\frac{\left(R_{1}-R_{O}\right)}{R_{O}} \times 100 \%,
$$

where $R$ is the sensor response (\%), $R_{1}$ is the voltage of the sensor when exposed to hexanal, and $R_{O}$ is the initial output voltage in the absence of hexanal. The testing was done by exposing hexanal with the concentration of $\sim 20 \mathrm{ppm}$, $\sim 100 \mathrm{ppm}, \sim 200 \mathrm{ppm}$, and $300 \mathrm{ppm}$.

\section{Results and Discussion}

3.1. FTIR Characterization. FTIR characterization was carried out to investigate the functional groups which exist in the chitosan film. The FTIR spectrum of the chitosan film is displayed in Figure 2. The peak which appeared at $3306.00 \mathrm{~cm}^{-1}$ is due to overlapping of $\mathrm{NH}_{2}$ and $\mathrm{OH}$ in chitosan film. The peak observed near $1634.97 \mathrm{~cm}^{-1}$ is corresponding to $\mathrm{C}=\mathrm{O}$ group in chitosan film [7]. Besides that, N-H group can be seen at the spectrum of $1576.97 \mathrm{~cm}^{-1}$. The absorption peak near $1408.36 \mathrm{~cm}^{-1}$ specifies the existence of $\mathrm{OH}$ group in chitosan film [17]. Meanwhile, the C-N group which represents the amino group in chitosan film can be seen at the peak near the region of $1374.06 \mathrm{~cm}^{-1}$ [7]. Finally, the absorption peak which appeared at $1060.80 \mathrm{~cm}^{-1}$ represents the existence of $\mathrm{C}-\mathrm{O}$ group in chitosan film [17]. Overall, chitosan film consists of $\mathrm{NH}_{2}, \mathrm{OH}, \mathrm{C}-\mathrm{N}, \mathrm{C}-\mathrm{O}$, and $\mathrm{C}=\mathrm{O}$ in its structure. The appearance of $\mathrm{NH}_{2}$ indicates that the domain functional group of chitosan for sensing activity exists in the film even after being processed into film. 


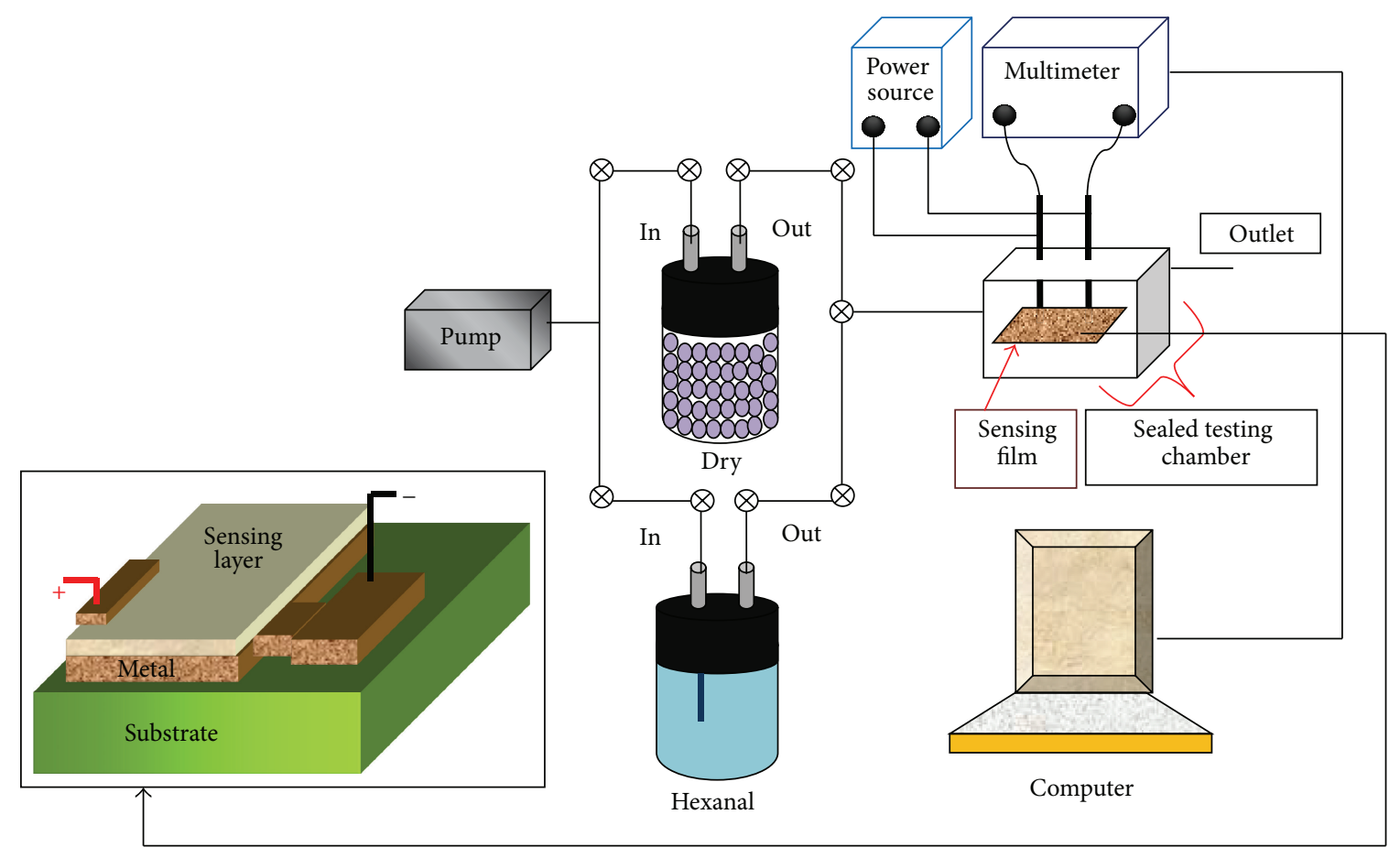

FIGURE 1: Electrical setup for hexanal detection.

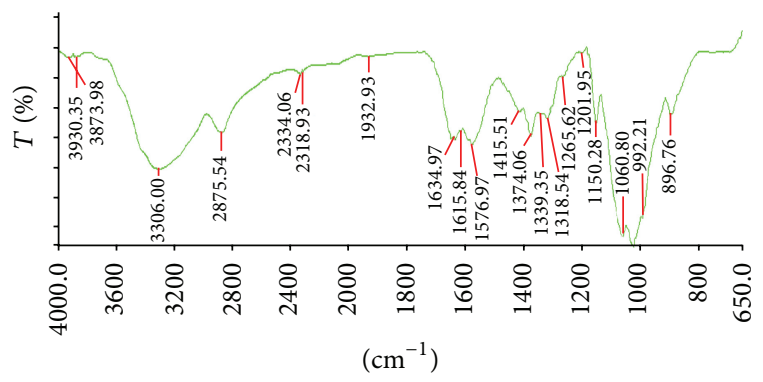

FIGURE 2: FTIR spectrum of chitosan film.

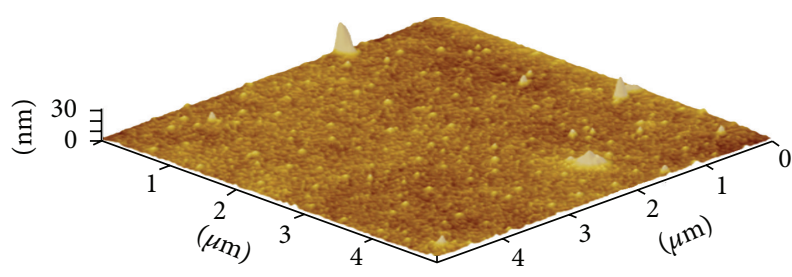

FIGURE 3: Surface of chitosan film sensor.

3.2. Atomic Force Microscopy (AFM). The surface structure of the chitosan film is presented in Figure 3. Chitosan film presented a homogeneous surface with a very low dispersion in the roughness profile with the RMS value of $1.140 \mathrm{~nm}$. This result was in accordance with research done by other researchers before [18]. Thus, it can be said that the film was deposited uniformly on the substrate without any agglomeration [15]. The strong bond of the films and substrate is

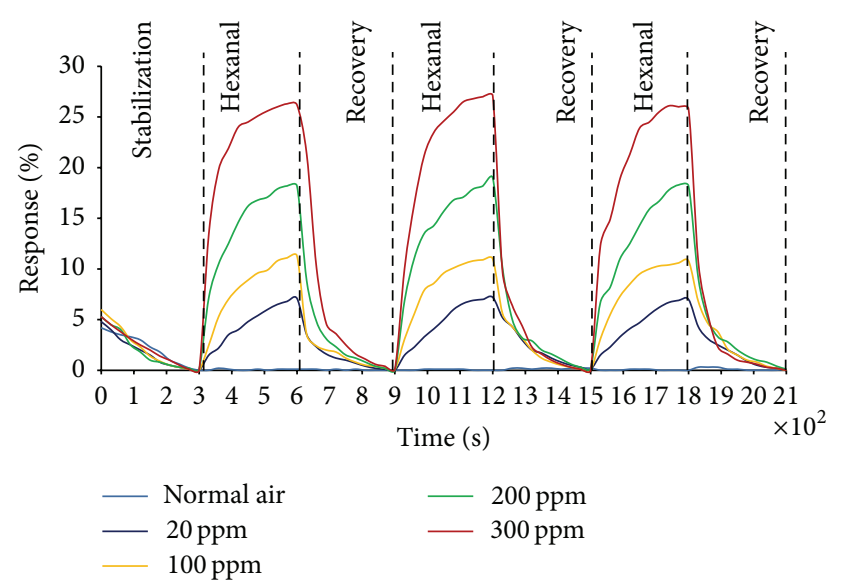

FIGURE 4: Response of the CFS toward hexanal gas.

very crucial to avoid swelling of the films during gas sensing measurement.

3.3. Hexanal Gas Sensing Studies. Figure 4 shows the response of chitosan film sensor (CFS) toward hexanal gas exposure, respectively. The purpose of this study is to investigate the sensing properties of the sensors toward hexanal gas including response of the sensor, response time, recovery time, repeatability, stability, lifetime, and selectivity of the CFS upon hexanal gas exposure.

3.3.1. Response. Response of the sensor is defined as the ability of the sensor to respond to various target molecules 


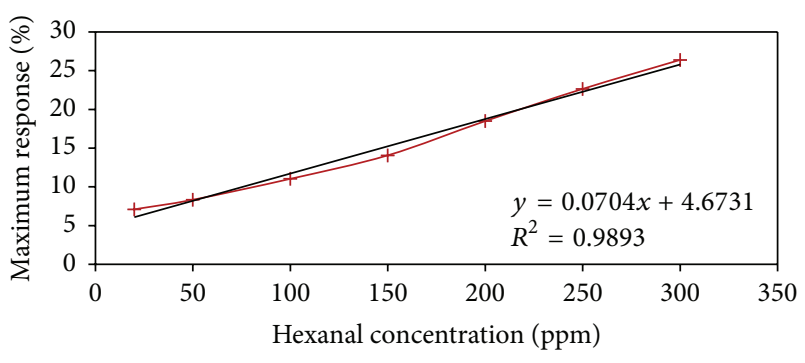

Figure 5: Calibration curve of the CFS toward hexanal gas.

[15]. In this present study, it was found that CFS has good response towards hexanal gas exposure. The response of the sensor increased with increasing gas concentration. It can be seen in Figure 5 that CFS has linear response towards gases indicated by the correlation value $\left(R^{2}\right)$ which is near to 1 . The CFS yielded maximum response values of approximately $\sim 7.10 \%, \sim 11.03 \%, \sim 18.46 \%$, and $\sim 26.36 \%$ when it was exposed to $20 \mathrm{ppm}, 100 \mathrm{ppm}, 200 \mathrm{ppm}$, and $300 \mathrm{ppm}$ of hexanal gas, respectively.

\subsubsection{Response Time and Recovery Time of CFS upon Hexanal} Gas Exposure. Initial response time is defined as the time taken for the sensor to sense the presence of target molecules on its surface [15]. The response time can also be defined as the time taken by a sensor to achieve $90 \%$ of total signal changes in the case of adsorption [19]. Meanwhile, recovery time is defined as the time needed for the sensor to reach $90 \%$ of the baseline value in the case of desorption [20]. The response time and recovery time of the CFS towards hexanal exposure are delineated in Figure 6. In this study, it can be seen that CFS started to respond in just 10 seconds after the gas was exposed. CFS took approximately 200 seconds to reach its $90 \%$ of the total signal change. As for recovery, the sensor started to recover in 10 seconds as well after the gas exposure was removed. It was observed that the recovery time of the sensor towards hexanal exposure was around 200 seconds.

3.3.3. Repeatability of CFS toward Hexanal Exposure. Repeatability is another important sensor property and it is defined by the International Organization for Standardization (ISO) as the repeatability conditions where independent test results are obtained with the same method on identical items in the same laboratory by the same operator using same equipment within interval time [21]. It can be seen that CFS has good repeatability as illustrated in Figure 7 where it does meet almost the same response for hexanal. From the results, it can be concluded that the repeatability of CSF has been achieved.

3.3.4. Stability of CFS toward Hexanal Exposure. Stability is defined as ability of sensor to exhibit constant sensor signal during measurement [14]. The sensor is considered as stable since there is no significant fluctuation in terms of response value during the exposure of the gas for response process or dry air for recovery process.

3.3.5. Lifetime of CFS Hexanal Exposure. Lifetime of the sensor is defined as the time period taken for the sensor to work with the same performance [22]. In this study, hexanal gas exposure was repeated using the same sensor for every one week interval. The result showed that CFS yielded almost the same response for 2 weeks as depicted in Figure 8. However, further investigation needs to be done in order to determine the optimum lifetime of the sensor.

3.3.6. Selectivity of CFS toward Different Gases. Selectivity of the sensor is known as the ability of the sensor to sense the various gas exposures. The response of the CFS to various gases was tested in order to study its selectivity properties. In this study, ammonia, acetone, and toluene were used to investigate the selectivity properties of the CFS. The results showed that CFS is selective to these gases as depicted in Figure 9. It can be seen that hexanal yielded the lowest response followed by toluene, acetone, and ammonia for CFS.

3.4. Sensing Mechanism of CFS toward Hexanal Gas. There are few steps which are involved in the sensing mechanism of chitosan film sensor towards hexanal gas. The explanation regarding the sensing mechanism is illustrated in detail in Figure 10 and in (2)-(3). This sensing phenomenon can be explained due to the electrons which consist in the sensing layer. The electrons in chitosan film sensor gained energy from the supplied input voltage. These electrons were then trapped by the oxygen from the air which contributes to the formation of chemisorbed oxygen such as $\mathrm{O}_{2}{ }^{-}$or $\mathrm{O}^{-}$as described as follows:

$$
\begin{aligned}
& \mathrm{O}_{2 \text { (gas) }} \longleftrightarrow \mathrm{O}_{2 \text { (adsorbed) }} \\
& \mathrm{O}_{2 \text { (adsorbed) }}+\mathrm{e}^{-} \longleftrightarrow \mathrm{O}_{2}^{-} \text {(adsorbed) }
\end{aligned}
$$

This chemisorbed oxygen which forms a barrier on the film surface contributes to the decline in the sensor response. Once the chemisorbed oxygen is fully occupied on the sensor surface by trapping all the electrons within the film, a situation called steady state will occur where the sensor yields almost constant response. The mechanism of the sensor started to change via introduction of gas on its surface. When hexanal gas is introduced on the surface of the chitosan film sensor (CFS), the gas molecules interact with the chemisorbed oxygen which is occupied on the sensor surface and the electrons tend to be released within the chitosan structure as explained as follows:

$$
\mathrm{C}_{6} \mathrm{H}_{12} \mathrm{O}+\mathrm{O}_{2}^{-}{ }^{-} \text {(adsorbed) } \longrightarrow \mathrm{C}_{6} \mathrm{H}_{12} \mathrm{O}_{2}+\mathrm{H}_{2} \mathrm{O}+\mathrm{e}^{-}
$$

From (4), it can be seen that the reaction will release electrons and water molecules. The released electrons become free electrons in the conduction band and induce the increment of the electrical response (\%) of the sensor. Meanwhile, the water molecules might form hydrogen bonding with the surface of the sensing layer. This bonding might enhance the 


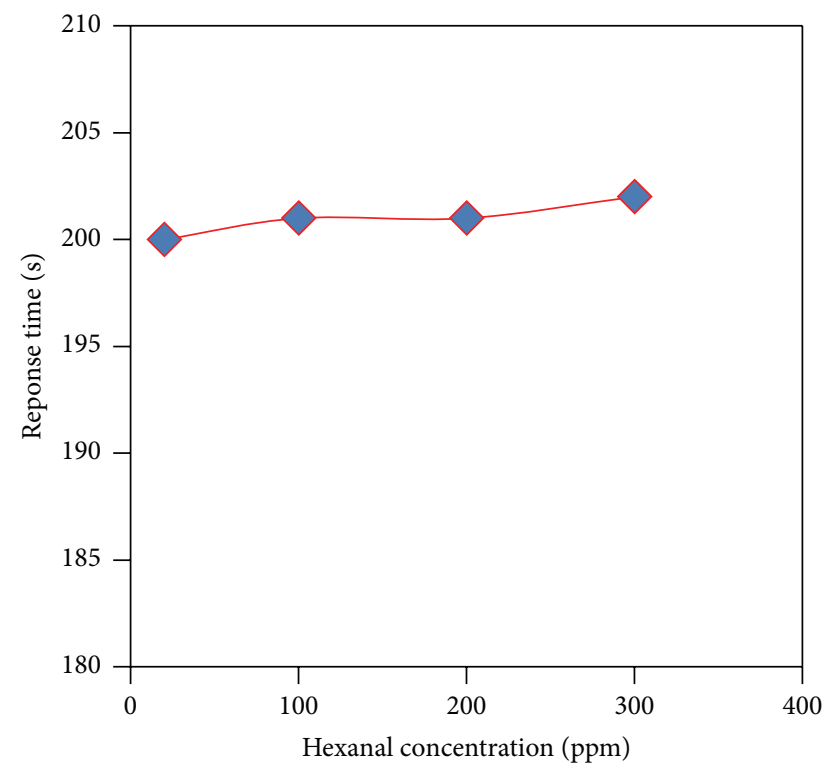

(a)

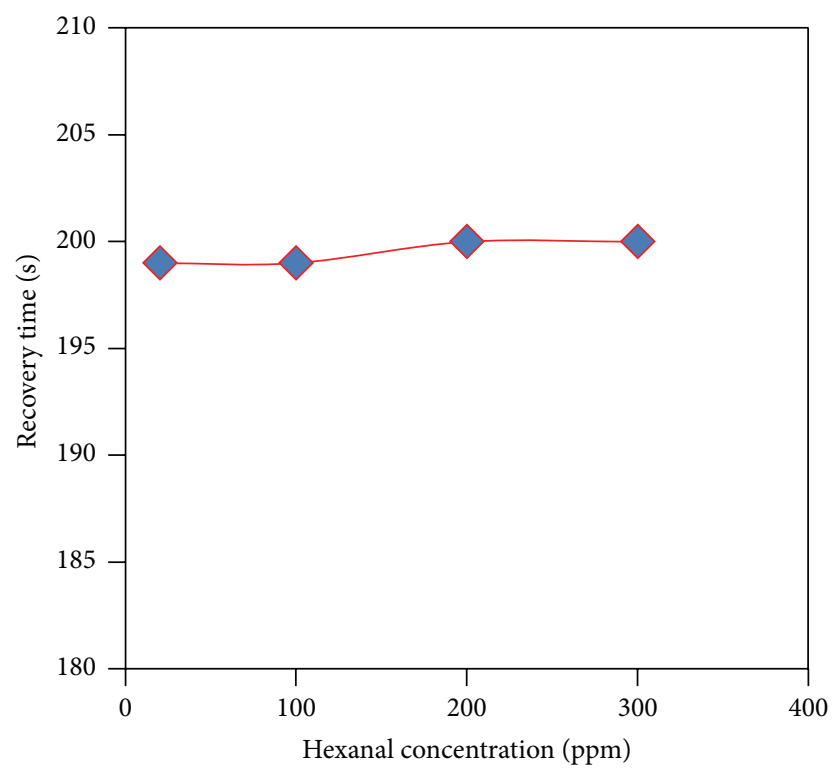

(b)

FIGURE 6: (a) Response time and (b) recovery time of the CFS toward various concentrations of hexanal gas.

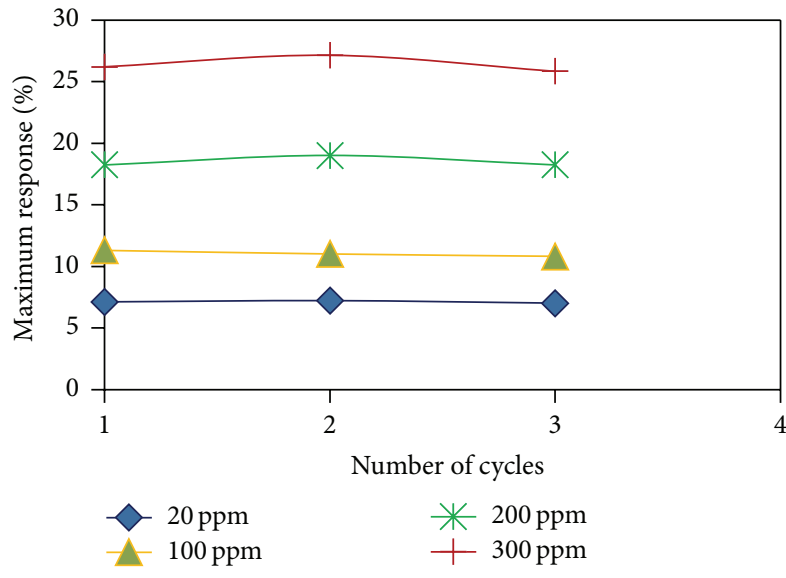

Figure 7: Repeatability of the CFS toward hexanal gas.

electron movements which contribute to increment of the response of the sensor as well.

\section{Conclusion}

In this study, chitosan based hexanal sensor was successfully developed using electrochemical deposition method. The sensor fulfils all the sensing properties of a reliable sensor including good response, recovery, repeatability, lifetime, and stability towards hexanal exposure. Besides that, this sensor exhibited good selectivity towards 3 other gases. Furthermore, the ability of the sensor to operate at room temperature with low energy consumption and low fabrication cost makes it become one of the reliable sensors which can be utilized

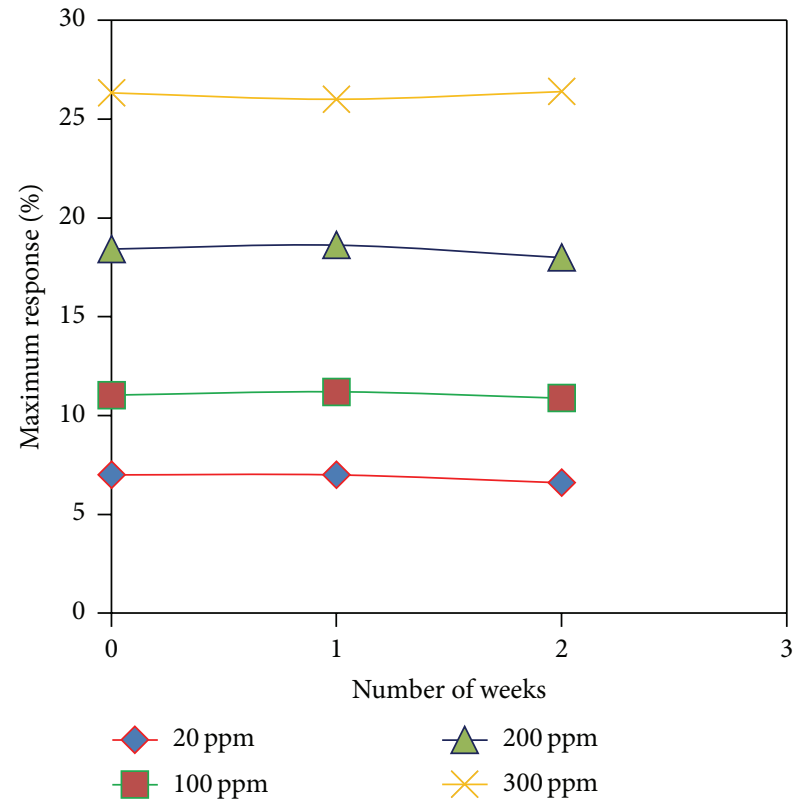

FIGURE 8: Lifetime of the CFS toward hexanal gas.

in various sectors especially in wood and dairy industries. However, further testing and investigation have to be carried out in order to improve the performance of the CFS.

\section{Conflict of Interests}

The authors declare that there is no conflict of interests regarding the publication of this paper. 


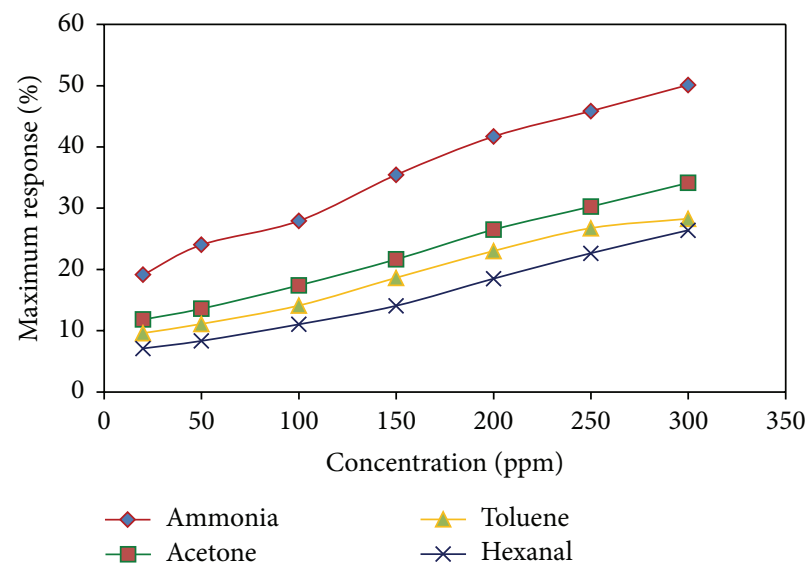

FIGURE 9: Selectivity of CFS toward ammonia, acetone, toluene, and hexanal exposure.
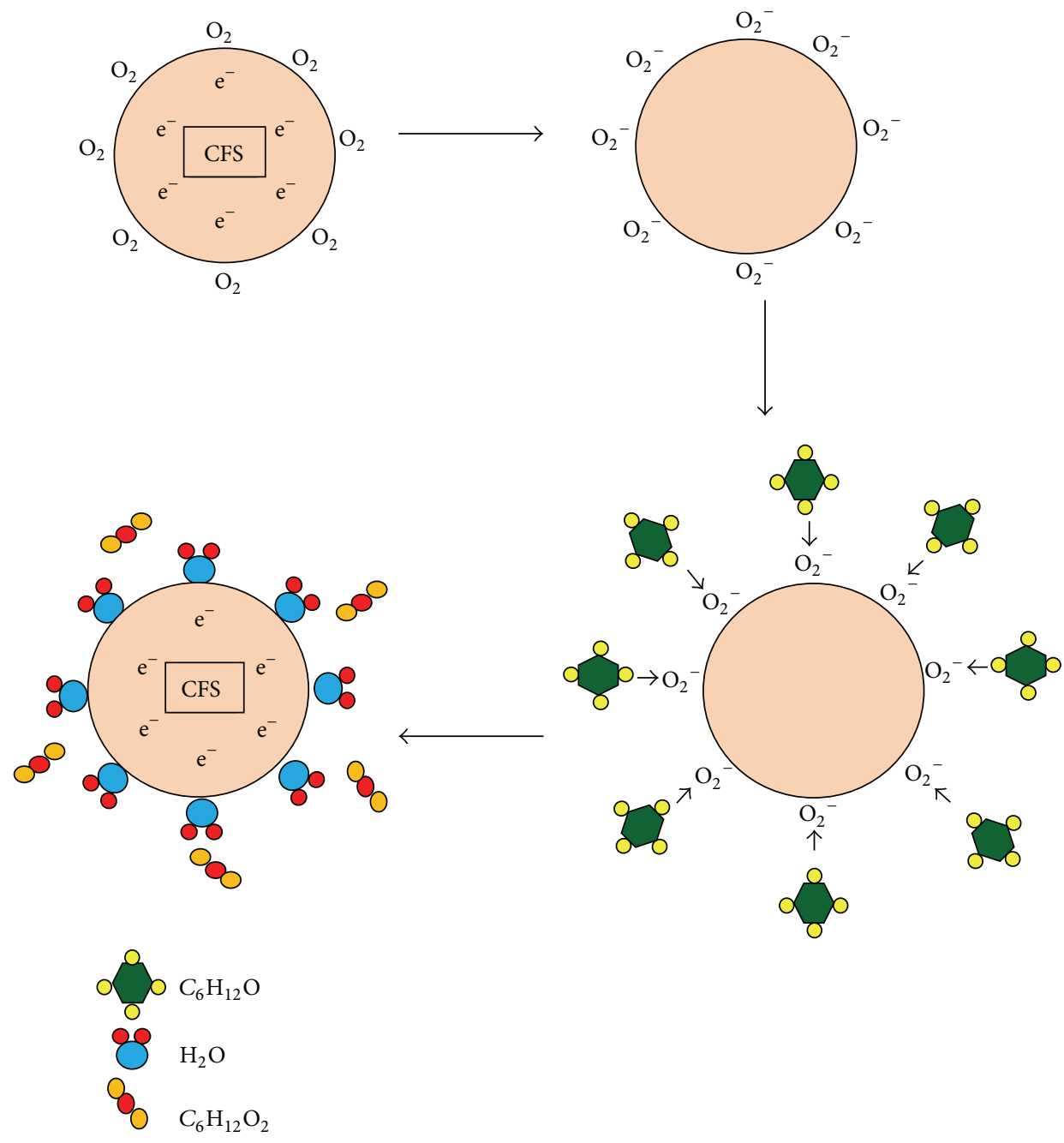

FIGURE 10: Sensing mechanism of CFS toward hexanal exposure. 


\section{Acknowledgments}

The authors would like to acknowledge the funding and support from the Fundamental Research Grant Scheme (FRGS) 9003-00346, Ministry of Higher Education, Malaysia. The authors thank School of Materials Engineering, UniMAP, for other supports.

\section{References}

[1] E. J. Montelius, Scientific Basis for Swedish Occupational Standards XXVII, 2006.

[2] M. Romeu-Nadal, J. L. Chávez-Servín, A. I. Castellote, M. Rivero, and M. C. López-Sabater, "Oxidation stability of the lipid fraction in milk powder formulas," Food Chemistry, vol. 100, no. 2, pp. 756-763, 2007.

[3] B. O. Galle, "Emission of hexanal and carbon monoxide from storage of wood pellets, a potential occupational and domestic health hazard," British Occupational Hygiene Society, vol. 48, no. 4, pp. 339-349, 2004.

[4] F. Ulberth and D. Roubicek, "Monitoring of oxidative deterioration of milk powder by headspace gas chromatography," International Dairy Journal, vol. 5, no. 6, pp. 523-531, 1995.

[5] M. L. Rodríguez-Méndez, J. Souto, R. de Saja, J. Martínez, and J. Antonio De Saja, "Lutetium bisphthalocyanine thin films as sensors for volatile organic components (VOCs) of aromas," Sensors and Actuators, B: Chemical, vol. 58, no. 1-3, pp. 544-551, 1999.

[6] K. Lee, K. Lee, V. Lau, K. Shin, and B.-K. Ju, "SWNT sensors for monitoring the oxidation of edible oils," Journal of Science and Technology, vol. 22, no. 4, pp. 239-243, 2013.

[7] S. Yalçinkaya, C. Demetgül, M. Timur, and N. Çolak, "Electrochemical synthesis and characterization of polypyrrole/chitosan composite on platinum electrode: its electrochemical and thermal behaviors," Carbohydrate Polymers, vol. 79, no. 4, pp. 908-913, 2010.

[8] I. Nainggolan, D. Shantini, T. I. Nasution, and M. N. Derman, "Role of metals content in spinach in enhancing the conductivity and optical band gap of chitosan films," Advances in Materials Science and Engineering, vol. 2015, Article ID 702815, 8 pages, 2015.

[9] D. S. Chandrasakaran, I. Nainggolan, T. Ikhsan, and N. Derman, "Ammonia gas sensor based on chitosan biopolymer," Material Science Forum, vol. 819, pp. 429-434, 2015.

[10] A. Bouvree, M. Castro, Y. Grohens, and M. Rinaudo, "Conductive polymer nano-bioComposites (CPC): chitosan-carbon nanoparticle a good candidate to design polar vapour sensors," Sensors and Actuators B: Chemical, vol. 138, no. 1, pp. 138-147, 2009.

[11] D. Feng, F. Wang, and Z. Chen, "Electrochemical glucose sensor based on one-step construction of gold nanoparticle-chitosan composite film," Sensors and Actuators, B: Chemical, vol. 138, no. 2, pp. 539-544, 2009.

[12] B. Kumar, J.-F. Feller, M. Castro, and J. Lu, "Conductive bioPolymer nano-Composites (CPC): chitosan-carbon nanotube transducers assembled via spray layer-by-layer for volatile organic compound sensing," Talanta, vol. 81, no. 3, pp. 908-915, 2010.

[13] R. Pauliukaite, M. E. Ghica, O. Fatibello-Filho, and C. M. A. Brett, "Electrochemical impedance studies of chitosan-modified electrodes for application in electrochemical sensors and biosensors," Electrochimica Acta, vol. 55, no. 21, pp. 6239-6247, 2010.

[14] W. Li, D. M. Jang, S. Y. An, D. Kim, S.-K. Hong, and H. Kim, "Polyaniline-chitosan nanocomposite: high performance hydrogen sensor from new principle," Sensors and Actuators, B: Chemical, vol. 160, no. 1, pp. 1020-1025, 2011.

[15] T. I. Nasution, I. Nainggolan, S. D. Hutagalung, K. R. Ahmad, and Z. A. Ahmad, "The sensing mechanism and detection of low concentration acetone using chitosan-based sensors," Sensors and Actuators, B: Chemical, vol. 177, pp. 522-528, 2013.

[16] P. Dykstra, J. Hao, S. T. Koev, G. F. Payne, L. Yu, and R. Ghodssi, "An optical MEMS sensor utilizing a chitosan film for catechol detection," Sensors and Actuators, B: Chemical, vol. 138, no. 1, pp. 64-70, 2009.

[17] M. R. Derrick, D. Stulik, and J. M. Landy, Infrared Spectroscopy in Conservation Science, Scientific Tools for Conservation, 1999.

[18] A. P. P. Praxedes, A. J. C. da Silva, R. C. da Silva et al., "Effects of UV irradiation on the wettability of chitosan films containing dansyl derivatives," Journal of Colloid and Interface Science, vol. 376, no. 1, pp. 255-261, 2012.

[19] X. Zhou, J. Liu, C. Wang et al., "Highly sensitive acetone gas sensor based on porous $\mathrm{ZnFe}_{2} \mathrm{O}_{4}$ nanospheres," Sensors and Actuators B: Chemical, vol. 206, pp. 577-583, 2015.

[20] R. Pandeeswari and B. G. Jeyaprakash, "High sensing response of $\beta-\mathrm{Ga}_{2} \mathrm{O}_{3}$ thin film towards ammonia vapours: influencing factors at room temperature," Sensors and Actuators, B: Chemical, vol. 195, pp. 206-214, 2014.

[21] R. P. Buck, E. Lindner, W. Kutner, and G. Inzelt, "Piezoelectric chemical sensors," Pure and Applied Chemistry, vol. 76, no. 6, pp. 1139-1160, 2004.

[22] D. K. Ashwal and S. K. Gupta, "Material, mechanism and fabrication," in Science and Technology of Chemiresistor Gas Sensor, chapter 2, pp. 1-380, 2007. 


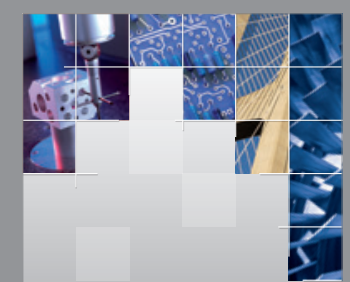

\section{Enfincering}
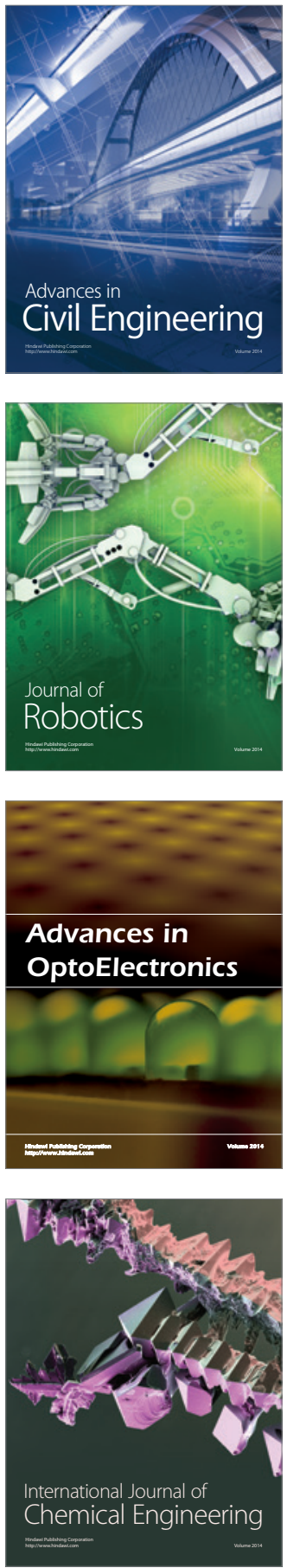

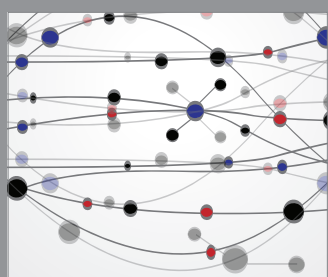

The Scientific World Journal

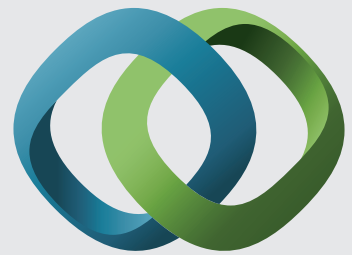

\section{Hindawi}

Submit your manuscripts at

http://www.hindawi.com
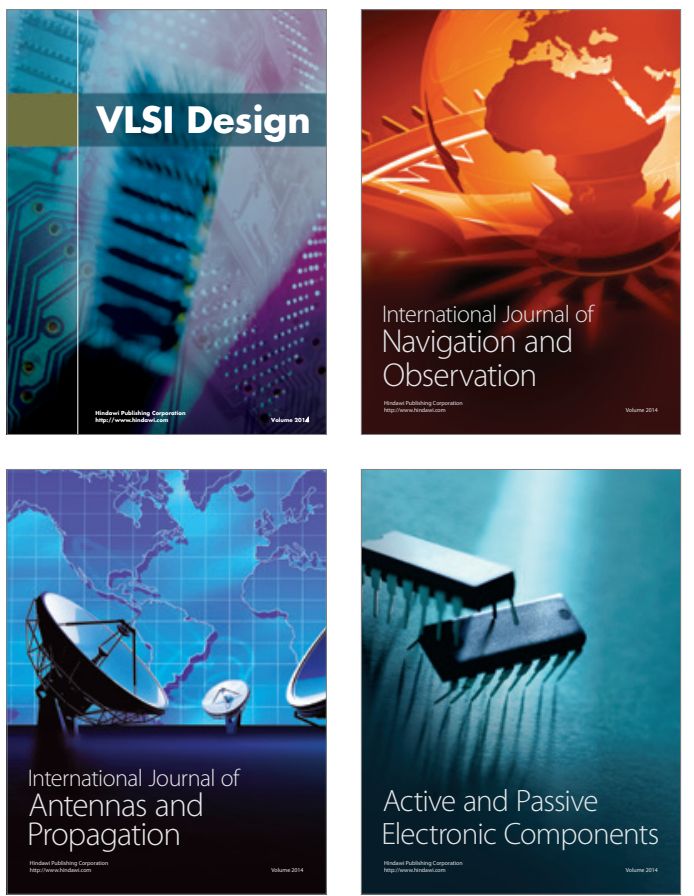
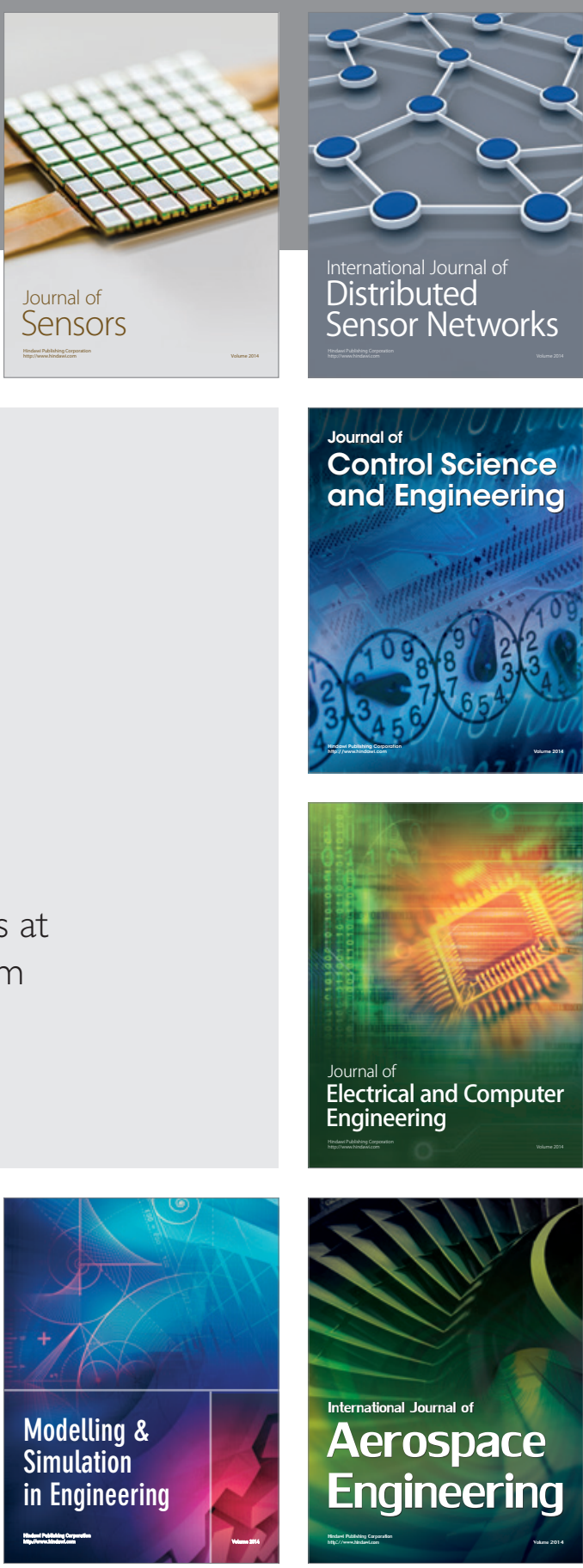

International Journal of

Distributed

Sensor Networks

Journal of

Control Science

and Engineering
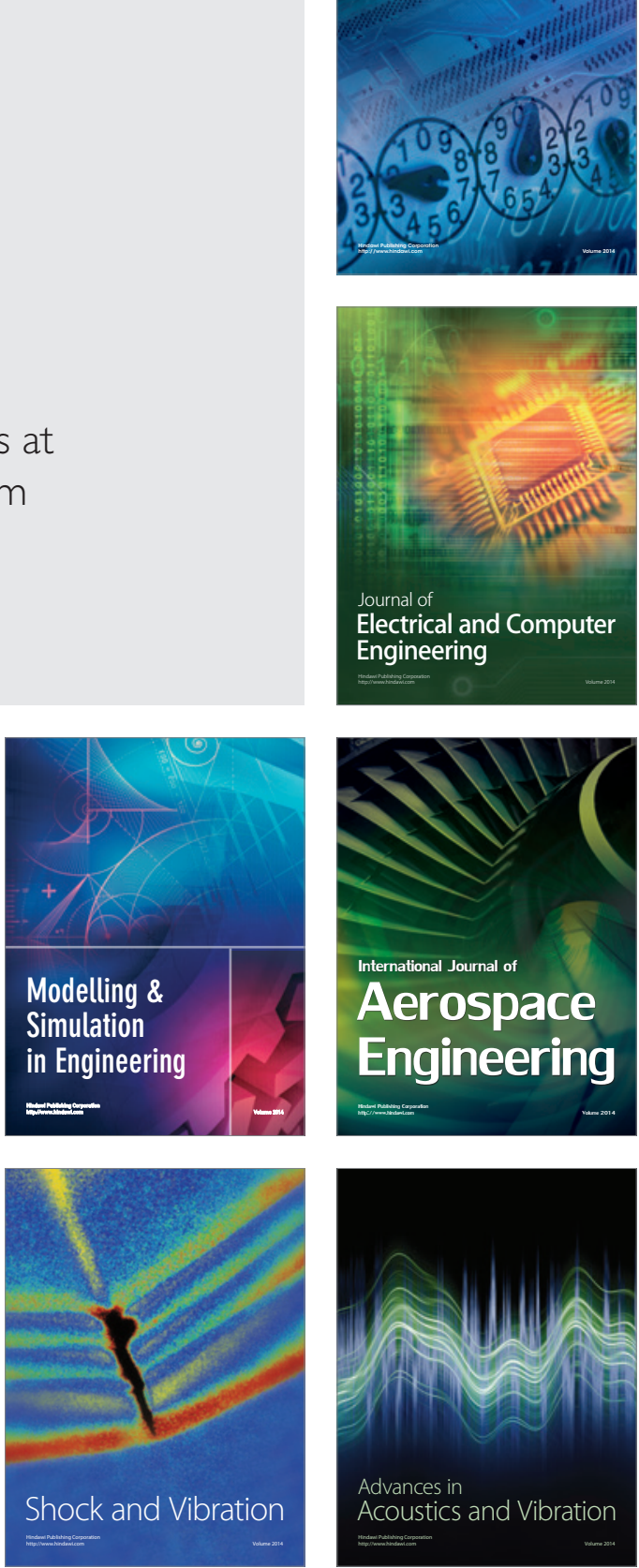